\title{
Segmentation, Skeletonization, and Branchpoint Matching - A Fully Automated Quantitative Evaluation of Human Intrathoracic Airway Trees
}

\author{
J. Tschirren ${ }^{1}$, K. Palágyi ${ }^{4}$, J. M. Reinhardt ${ }^{2}$, E. A. Hoffman ${ }^{3,2}$, and M. Sonka ${ }^{1}$ \\ 1 Department of Electrical and Computer Engineering \\ 2 Department of Biomedical Engineering \\ 3 Department of Radiology \\ The University of Iowa, Iowa City, IA 52242, USA \\ 4 Department of Applied Informatics, University of Szeged, Hungary
}

\begin{abstract}
Modern multislice X-ray CT scanners provide high-resolution volumetric image data containing a wealth of structural and functional information. The size of the volumes makes it more and more difficult for human observers to visually evaluate their contents. Similar to other areas of medical image analysis, highly automated extraction and quantitative assessment of volumetric data is increasingly important in pulmonary physiology, diagnosis, and treatment. We present a method for a fully automated segmentation of a human airway tree, its skeletonization, identification of airway branches and branchpoints, as well as a method for matching the airway trees, branches, and branchpoints for the same subject over time and across subjects. The validation of our method shows a high correlation between the automatically obtained results and reference data provided by human observers.
\end{abstract}

\section{Introduction}

Quantitative assessment of intrathoracic airway trees is critically important for objective evaluation of bronchial tree structure and function. Several approaches to three-dimensional reconstruction of the airway tree have been developed in the past. None of them, however, allows direct comparison of airway trees across and within subjects. Functional understanding of pulmonary anatomy as well as the natural course of respiratory diseases like asthma, emphysema, cystic fibrosis, and many others is limited by our inability to repeatedly evaluate the same region of the lungs time after time and perform accurate and reliable positionally corresponding measurements. Consequently, quantitative analysis of disease status and its progression and regression, as well as longitudinal physiologic and functional analyses are impossible. In this paper, we describe an integrated approach to quantitative analysis of intrathoracic airway trees and inter-tree matching using high-resolution volumetric computed tomography (CT) images.

T. Dohi and R. Kikinis (Eds.): MICCAI 2002, LNCS 2489, pp. 12 2002.

(C) Springer-Verlag Berlin Heidelberg 2002 


\section{Methods}

The reported system consists of three main blocks: airway tree segmentation, skeletonization and branchpoint localization, and branchpoint matching. Each of these blocks is described separately in the following subsections.

\subsection{Airway tree segmentation}

The airway segmentation takes advantage of the relatively high contrast in $\mathrm{CT}$ images between the center of an airway and the airway wall. A seeded region growing is employed starting with an automatically identified seedpoint within the trachea. New voxels are added to the region if they have a similar X-ray density as a neighbor voxel that already belongs to the region. The similarity measure is designed so that the region growing can overcome subtle gray level changes (like for example caused by beam-hardening). On the other hand a "leaking" into the surrounding lung tissue has to be avoided. This is realized by setting an upper limit of allowed difference in gray value for two neighboring voxels. Our region growing algorithm utilizes a breadth-first search [1], which allows a fast and memory-friendly implementation. After airway segmentation, a binary subvolume is formed that represents the extracted airway tree.

\subsection{Skeletonization}

The binary airway tree formed in the previous step is skeletonized to identify the three-dimensional centerlines of individual branches and to determine the branchpoint locations. A sequential 3D thinning algorithm reported by Palágyi et al. [2] was customized for our application. To obtain the skeleton, a thinning function deletes border voxels that can be removed without changing the topology of the tree. This thinning step is applied repeatedly until no more points can be deleted. The thinning is performed symmetrically and the resulting skeleton is guaranteed to lie in the middle of the cylindrically shaped airway segments.

After completion of the thinning step, the skeleton is smoothed, false branches pruned, the location of the branchpoints identified, and the complete tree converted into a graph structure using an adjacency list representation. Fig. 1 shows a close-up view of a skeleton produced by the algorithm. Skeleton branchpoints are identified as skeleton points with more than two neighboring skeleton points.

\subsection{Branchpoint matching}

The goal of branchpoint matching is to find anatomically corresponding branchpoints in two different airway trees. Two types of matching are of utmost interest: intra-subject and inter-subject matching. In the first case, trees coming from different scans of the same subject are matched. In the second case, two or more trees are matched originating from different subjects. The latter case only allows matching of the primary branchpoints (the first three or four generations). These 

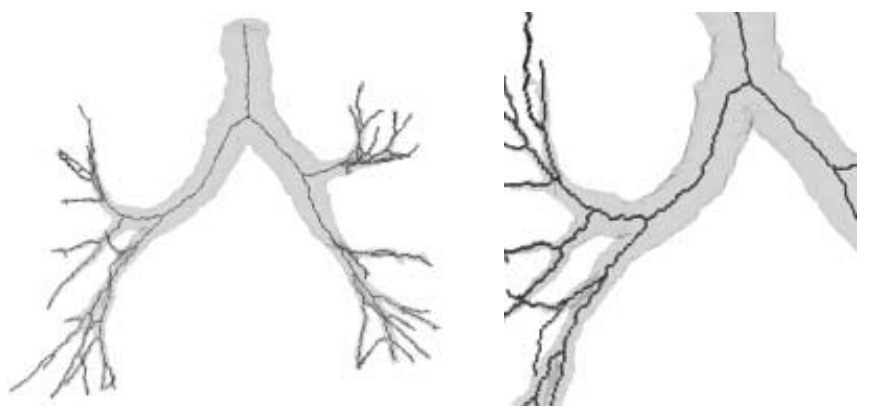

Fig. 1. Example of segmentation and skeletonization applied on an airway tree phantom.

primary branchpoints are frequently (although not universally) identical among humans. The branching pattern of higher airway generations varies from subject to subject, much like fingerprints do.

In the mathematical sense, an airway tree is a graph (rooted tree). The branchpoints correspond to vertices and the airway segments correspond to graph edges. There are many graph-theoretic approaches to graph matching. A widely used method for matching hierarchical relational structures is to map them onto an association graph and then find its maximum clique [3], with many variations existing $[4,5]$. To the best of our knowledge, only one application of the method was employed for matching airway trees [6].

A disadvantage of finding the maximum clique is its NP-completeness [7]. This means that for all but small graphs, an exhaustive search is not feasible. There are two basic ways of decreasing the computational complexity: minimizing the overall problem size or splitting the problem into several smaller subproblems. Our method uses both of these strategies.

Terminal branches that are shorter than a predefined length are mostly spurious (caused by inaccuracies in the segmentation and skeletonization processes) and are pruned out of the tree in the late stages of the skeletonization process. Additionally, the major vertices (branchpoints) are identified. A vertex is considered to be major if it has at least $N$ vertices hierarchically underneath it, and if these vertices have a spatial extent that exceeds a predefined threshold. The spatial extent is defined as the maximum of the three differences $x_{\max }-x_{\min }$, $y_{\max }-y_{\min }$, and $z_{\max }-z_{\min }$. Next, the two trees undergo a rigid registration, using the major branchpoints as landmark points. The major branchpoints are matched using an association graph. After that, a separate association graph is created for every subtree starting from a set of matched major branchpoints. When creating the association graphs for the sub-trees, only vertex-pairs that lie relatively close to each other are considered. This reduces the size of the association graph. Edges are added to the association graph based on the topological and geometrical distances, inheritance relationships, and geometrical length and directions. For all of these measures tolerances are allowed. For the topological 
distance, a tolerance of \pm 2 segments is allowed. A parent-child and a childparent relationship are regarded equivalent if the geometrical distance between the two branchpoints does not exceed $2 \mathrm{~mm}$ in both trees. This introduces tolerance for cases where two branches are very close to each other, and - due to tolerances in segmentation and skeletonization - the order of two branchpoints is swapped for the two trees. For the length and angles of segments, tolerances of $\pm 20 \%$ and \pm 0.2 radians are allowed, respectively. Allowing for these tolerances introduces robustness against false branches and missing branches. In a final step, the maximum clique is found for every association graph.
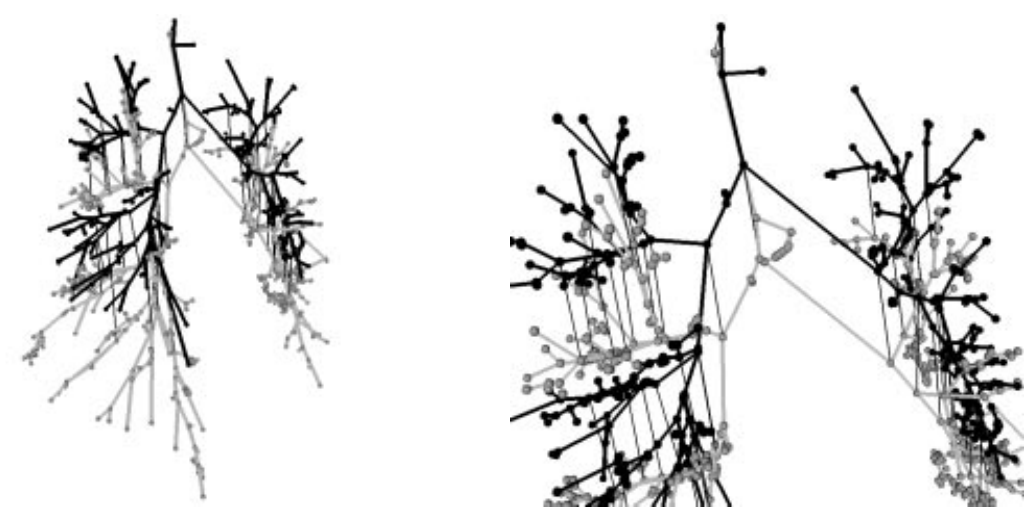

Fig. 2. Result of branchpoint matching for in vivo scan (TLC and FRC), total view and detail view of same matching. The two trees are shown in bold black and bold gray, the matchings are represented by fine black lines.

\section{Experimental Methods}

To test the method, CT scans of two different physical phantoms and in vivo scans of the human airway trees were used.

\subsection{Data}

Two different phantoms were available. The first phantom is a hollow rigid plastic phantom (Fig. 3 a), made by a rapid prototyping machine. The phantoms geometry is based on real human data. Consequently, a human-like airway tree with parameters known to a high degree of accuracy is available. This phantom consists of about 100 airway tree branches with about 50 branchpoints (not counting the terminal points of airway segments). The second phantom is a hollow rubber phantom (Fig. 3b) made from a human airway tree cast. This second phantom is more complex, consisting of about 400 branches and 200 branchpoints. The 
rubber phantom was scanned in a Perspex container filled with potato flakes to resemble texture of surrounding pulmonary parenchyma. Since this rubber phantom was not built using a numerical rapid prototyping approach and it is not rigid, exact branchpoint locations were not known.

The rigid phantom was CT-scanned at three different angles $\left(0^{\circ}, 10^{\circ}\right.$, and $25^{\circ}$ ) by rotating it on the scanner table (rotation around one axis). The rubber phantom was scanned twice. It was rotated in a similar way as the rigid phantom. The rotation angle was $8^{\circ}$ in this case. The pixel size was $0.49 \times 0.49 \times 0.60 \mathrm{~mm}^{3}$ for the rigid phantom and $0.68 \times 0.68 \times 0.60 \mathrm{~mm}^{3}$ for the rubber phantom. The volume sizes were $512 \times 512 \times 500-600$ voxels.

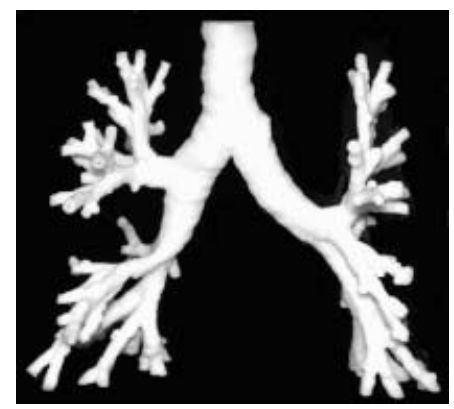

(a)

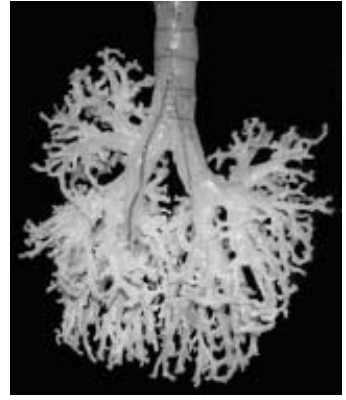

(b)

Fig. 3. Phantoms. a) Rigid phantom, b) Rubber phantom. In both phantoms, all the airway segments are hollow.

Two scans were available for each of 18 in vivo subjects for a total of 36 volumetric high resolution in vivo CT scans. For each subject, a scan close to total lung capacity (TLC) was acquired (at $85 \%$ lung volume), and a scan close to functional residual capacity (FRC) was acquired (at $55 \%$ lung volume). All in vivo scans have a nearly isotropic resolution of $0.7 \times 0.7 \times 0.6 \mathrm{~mm}^{3}$ and consist of 500-600 image slices, $512 \times 512$ pixels each. In two of these $18 \mathrm{CT}$ data pairs (in 4 volumes, two from a diseased and two from a normal subject), branchpoints were manually identified by human observers and were used for quantitative validation.

\subsection{Validation indices}

The validation was done in two parts. First, the reproducibility of the segmentation and skeletonization was tested. Next, the accuracy of the branchpoint matching was examined.

The reproducibility of the segmentation and skeletonization was measured by comparing the lengths of corresponding airway segments between the different scans of the two phantoms. 
The accuracy of the branchpoint matching was measured by comparing the results obtained using the automated method with the results of manual matching. The manual matching was done separately and independently by six different observers. A matched pair of branchpoints was only included in the independent standard if it was matched by a majority of human observers involved.

\section{Results}

Our method above was successfully applied to all 5 phantom and 36 human datasets. In all cases, the method generated reliable trees, well-positioned skeletons and branchpoints, and provided consistent intra-subject matches. Quantitative validation results are reported below. Fig. 4 gives comparison of airway segment lengths. The p-values are calculated by analysis of variance (ANOVA), using an F-statistic, with the null hypothesis that the mean values are equal. The means and standard deviations for the segment length differences were:

$\begin{array}{lll}\text { Rigid phantom, } 0^{\circ} \text { versus } 10^{\circ}: & \mu_{1}=0.03 \mathrm{~mm} & \sigma_{1}=0.86 \mathrm{~mm} \\ \text { Rigid phantom, } 10^{\circ} \text { versus } 25^{\circ}: & \mu_{1}=-0.07 \mathrm{~mm} & \sigma_{1}=2.45 \mathrm{~mm} \\ \text { Rigid phantom, } 0^{\circ} \text { versus } 25^{\circ}: & \mu_{2}=-0.31 \mathrm{~mm} & \sigma_{2}=1.96 \mathrm{~mm} \\ \text { Rubber phantom, } 0^{\circ} \text { versus } 8^{\circ}: & \mu_{1}=0.24 \mathrm{~mm} & \sigma_{1}=1.04 \mathrm{~mm}\end{array}$

Table 1. Results for accuracy assessment of branchpoint matching.

\begin{tabular}{llll}
\hline & $\begin{array}{l}\text { RIGID PHANTOM } \\
0^{\circ} \text { Vs. } 10^{\circ}\end{array}$ & $\begin{array}{l}\text { IN VIVO } \\
\text { NORMAL }\end{array}$ & $\begin{array}{l}\text { IN VIVE } \\
\text { DISEASED }\end{array}$ \\
\hline $\begin{array}{l}\text { Correct matches: computer- } \\
\text { determined vs. independent }\end{array}$ & $38 / 39(97 \%)$ & $11 / 13(85 \%)$ & $17 / 19(89 \%)$ \\
standard & & & \\
Wrong matches & 0 & 1 & 0 \\
Missing matches & 1 & 1 & 2 \\
Total computer matches & 47 & 46 & 31 \\
\hline
\end{tabular}

Table 1 lists the results for the branchpoint matching. The segmentation, skeletonization, and matching processes execute very fast on a $1.2 \mathrm{GHz} \mathrm{AMD}$ Athlon based Linux system. For an image volume containing $512 \times 512 \times 524$ voxels, the segmentation step finishes in less than one second, the complete skeletonization, smoothing, and graph-generation process executes in about 48 seconds, and matching of two trees containing 150-200 branchpoints each requires one to two seconds. Consequently, a pair of trees can be analyzed and matched in about 100 seconds using our moderate-speed hardware. 

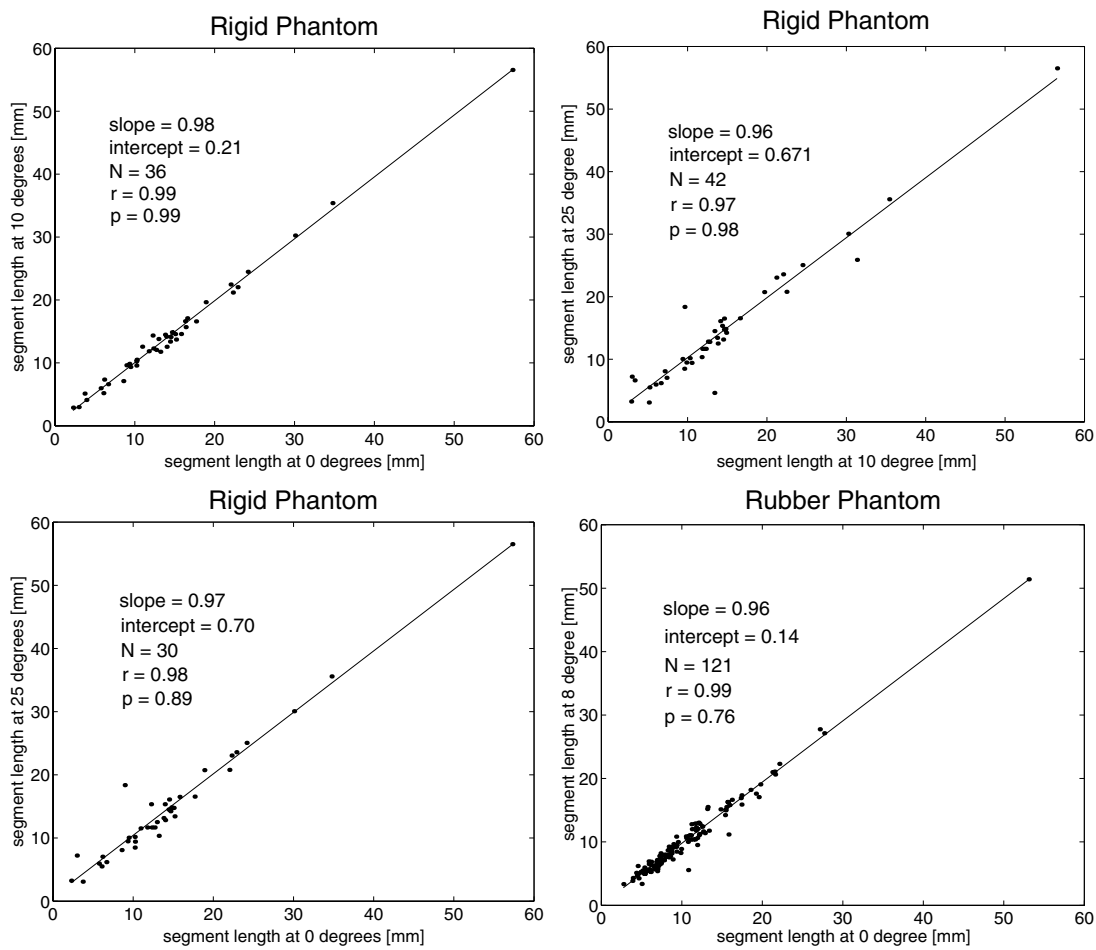

Fig. 4. Segment length comparison for rigid phantom and rubber phantom.

\section{Discussion}

The comparison of segment lengths as determined in phantoms showed high correlation between the reference data and the computer-determined data (Fig. 4). Agreement between segment lengths identified in the $0^{\circ}$ and $10^{\circ}$ rotated phantoms and for the $10^{\circ}$ and $25^{\circ}$ rotated phantoms was very good. For $0^{\circ}$ and $25^{\circ}$, somewhat larger differences between the lengths were observed. This is mainly caused by a few outliers likely to be associated with the relatively large change of the CT scanning conditions and is not practically important as $25^{\circ}$ differences between long-axis orientations of human subjects in a CT scanner is unlikely.

The comparison of computer-matched branchpoints and hand-matched branchpoints shows a high matching rate in the phantom cases $(97 \%)$, as well as in the human data $(85-89 \%)$. Notice that the human data contained a relatively high number of non-matching branches in the pairs of matched TLC and FRC datasets. Indeed, there is a considerable difference in the number of branches and in the identifiable parts of the tree-structures between FRC and TLC scans due to changes of lung volume and consequently lung geometry.

When comparing the matches identified manually and automatically, it is important to distinguish between missing and extra matches. Comparing be- 
tween these two classes only, a missing match is preferred over an extra match since no incorrect information is introduced. As can be seen in Table 1, only a single incorrect extra match was observed in the tested in vivo datasets. At the same time, a total of only four missing matches occurred - an encouraging sign considering that 77 correct matches were identified overall in the in vivo datasets and additional correct matches were found using the computer approach that were not identified manually.

The current implementation is not free of several shortcomings. The segmentation step is currently limited to the first 6 to 8 generations of airway tree segments. While substantially better than any of our previously reported approaches, additional improvements are under development. The branchpoint matching process is under review with a goal to avoid the small number of mismatches present in the current study. Needless to say, additional datasets are manually analyzed by human observers to form a larger and more representative set of independent standard data for future validation studies.

\section{Conclusion}

We presented an approach that allows reliable segmentation, skeletonization, and branchpoint matching in human airway trees. When tested in two kinds of physical phantoms derived from casts of human airway trees and in 36 invivo acquired airway trees of normal subjects as well as in those suffering from various pulmonary diseases, the method's performance was incomparably faster than manual analysis and yielded close-to-identical results.

\section{Acknowledgements}

This work was supported in part by the NIH grant HL-064368.

\section{References}

1. J. Silvela and J. Portillo, "Breadth-first search and its application to image processing problems," IEEE Transactions on Image Processing, vol. 10, pp. 1194-1199, 8 2001.

2. K. Palágyi, E. Sorantin, E. Balogh, A. Kuba, C. Halmai, B. Erdohelyi, and K. Hausegger, "A Sequential 3D Thinning Algorithm and its Medical Applications," in 17th Int. Conf. Information Processing in Medical Imaging, IPMI 2001, Davis, CA, USA. Lecture Notes in Computer Science 2082, pp. 409-415, 2001.

3. A. P. Ambler, H. G. Barrow, C. M. Brown, R. M. Burstall, and R. J. Popplestone, "A versatile computer-controlled assembly system," in Proceedings of International Joint Conference on Artificial Intelligence, pp. 298-307, 1973.

4. D. H. Ballard and C. M. Brown, Computer Vision. Prentice Hall PTR, 1982.

5. M. Pelillo, K. Siddiqi, and S. W. Zucker, "Matching hierarchical structures using association graphs," IEEE Transactions on Pattern Analysis and Machine Intelligence, vol. 21, pp. 1105-1120, 111999.

6. Y. Park, Registration of linear structures in 3-D medical images. PhD thesis, Osaka University, Japan. Department of Informatics and Mathematical Science., 12002.

7. T. H. Cormen, C. E. Leiserson, and R. L. Rivest, Introduction to Algorithms. MIT Press, 1990. 